\title{
OCEANIC LIDAR: THEORY AND EXPERIMENT
}

\author{
Dong Liu ${ }^{1, *}$, Yudi Zhou ${ }^{1, \#, ~ Q u n ~ L i u ̈ ~}{ }^{1}$, Weibiao Chen ${ }^{2}$, Aleksey Malinka ${ }^{3}$, Bing Han ${ }^{4}$, Zhihua Mao ${ }^{5}$, \\ Peituo Xu ${ }^{1}$, Zhipeng Liu ${ }^{1}$, Xiaoyu Cui ${ }^{1}$, Xiaobin Wang ${ }^{1}$, Haochi Che ${ }^{6}$, Peng Chen $^{5}$, Qingjun Song ${ }^{7}$, \\ Xiaolei $\mathrm{Zhu}^{2}$, Chengfeng Le ${ }^{8}$, Chong Liu ${ }^{1}$ \\ ${ }^{I}$ State Key Laboratory of Modern Optical Instrumentation, College of Optical Science and Engineering, \\ Zhejiang University, Hangzhou 310027, China \\ ${ }^{2}$ Shanghai Institute of Optics and Fine Mechanics, Chinese Academy of Sciences, Shanghai 201800, \\ China \\ ${ }^{3}$ Institute of Physics, National Academy of Sciences of Belarus, Pr. Nezavisimosti 68-2, Minsk 220072, \\ Belarus \\ ${ }^{4}$ National Ocean Technology Center, Tianjin 300112, China \\ ${ }^{5}$ The Second Institute of Oceanography, State Oceanic Administration, Hangzhou 310012, China \\ ${ }^{6}$ Department of Physics, University of Oxford, Oxford OXI 3PU, UK \\ ${ }^{7}$ National Satellite Ocean Application Service, Beijing 100081, China \\ ${ }^{8}$ College of Ocean, Zhejiang University, Hangzhou 310027, China \\ Email:*liudongopt@,zju.edu.cn"zhouyudi@zju.edu.cn
}

\begin{abstract}
Study on the upper ocean is of great significance to the global climate change and carbon cycle. Lidar can be used to effectively detect depthresolved optical properties of the ocean. However, both theory and experiment of oceanic lidar are limited by complex multiple scattering. Several progresses by Zhejiang University will be illustrated in this paper: 1) a polarized lidar system was developed, and a Monte Carlo model and a radiative transfer model were established (Zhou, et al. remote sensing, 2019; Zhou, et al. Journal of remote sensing, 2019; Xu, et al. and Liu, et al. Journal of remote sensing, 2019); 2) Cross validations are demonstrated to verify the availability of the lidar system and models (Liu, et al. IEEE TGRS, 2019); 3) phase function effects on backscatter and attenuation are studied considering multiple scattering, respectively (Liu, et al. Optics Express, 2019). Oceanic lidar is proven to have great potential in marine studies.
\end{abstract}

\section{INTRODUCTION}

Study on the upper ocean is of great significance to the global climate change and carbon cycle. The ocean color remote sensing based on the airplane or satellite, like SeaWiFS, is able to collect the global data over a long term efficiently. Nevertheless, the limited information about the depth and the dependence on the natural light partly restrict its applications. So far, the oceanographic lidar, one of the active remote sensing methods, has been employed in detecting fisheries, phytoplankton layers and internal waves, etc., in the upper ocean [1-3].

In general, interpretation of lidar signals is not difficult under the well-known single-scattering approximation. However, in dense media, such as clouds, seawater, etc., the preceding and following events are accompanied by strong multiple scattering [3]. A portion of photons that are lost in the scattering events could eventually contribute to the lidar signals through multiple scattering. As a result, large errors could be introduced into the single-scattering approximation. Therefore, it is important to quantify and analyze lidar signals with multiple scattering.

Several progresses by Zhejiang University will be illustrated in this paper: 1) a polarized lidar system was developed, and a Monte Carlo model and a radiative transfer model were established; 2) Cross validations are demonstrated to verify the availability of the lidar system and models; 3) phase function effects on backscatter and attenuation are studied considering multiple scattering, respectively.

\section{METHODOLOGY}

\subsection{The lidar system}

The developed shipborne oceanic lidar transmitted a laser pulse into the seawater and detected the lidar returns for the retrieval of the seawater optical properties, as shown in Fig. 1. The transmitter included a frequency-doubling Qswitched Nd:YAG pulsed laser at $532 \mathrm{~nm}$, with a 
single pulse energy of $5 \mathrm{~mJ}$, a pulse width of $10 \mathrm{~ns}$ and a repetition frequency of $10 \mathrm{~Hz}$.

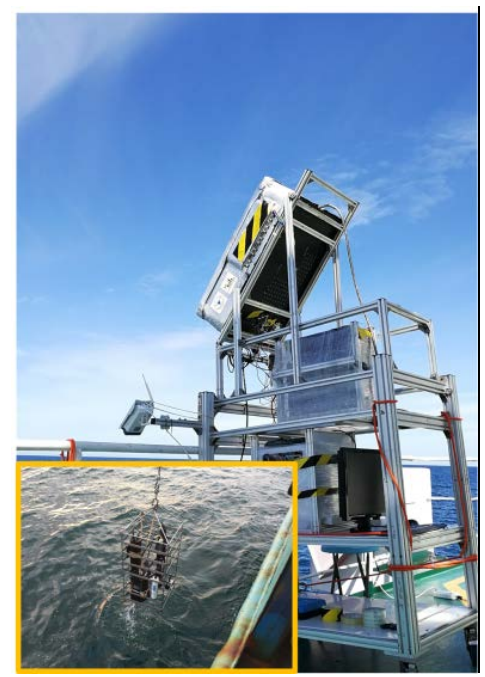

Fig. 1. The lidar field experiment in the Yellow Sea. Lidar system mounted on the fore deck of the ship and in situ instruments going into the water.

\subsection{Monte Carlo models}

The standard MC and semianalytic MC models are employed to provide MC-simulated results. The basic principle of the MC simulation is to treat photons as classical particles [4] and simulate the trajectories of a number of photons to measure the relevant information. The standard $\mathrm{MC}$ algorithm refers to the method described in [4]. The semianalytic MC algorithm refers to the method illustrated in [5], where an analytical estimate is calculated for the possibility of the collection of scattered photons at certain points.
The semianalytic MC algorithm greatly improves the calculation efficiency compared with the standard MC algorithm [6].

\subsection{Analytical model}

The analytical model employed in this paper follows the work of Katsev [7] and Malinka [8]. Seawater has very sharp forward peaks in its phase function that make the probability of scattering into the near-forward directions much larger than that of scattering into the backward hemisphere. If the optical thickness of the media is not too large, it is assumed that the trajectories that contribute to lidar signals primarily consist of single backscattering and small-angle forward multiple scattering on the outgoing and returning legs [7], namely the QSA approximation, which forms the foundation of the analytical model [6].

\section{RESULTS}

\subsection{Cross validation}

The lidar signals calculated by the analytical model, semianalytic MC algorithm and standard $\mathrm{MC}$ algorithm are shown in Fig. 2. The effects of height, FOV and water type are shown in Fig. 2 (a)-(c), respectively. The semianalytic MC and standard MC algorithms are simplified as "Semi $M C$ " and "Stan MC", respectively, in each legend. To give a more explicit picture, the lidar signals are normalized by their maximums, and the dynamic ranges are set to 4 orders of magnitude. In Fig. 2, the results of the three algorithms agree very well at different conditions [6].
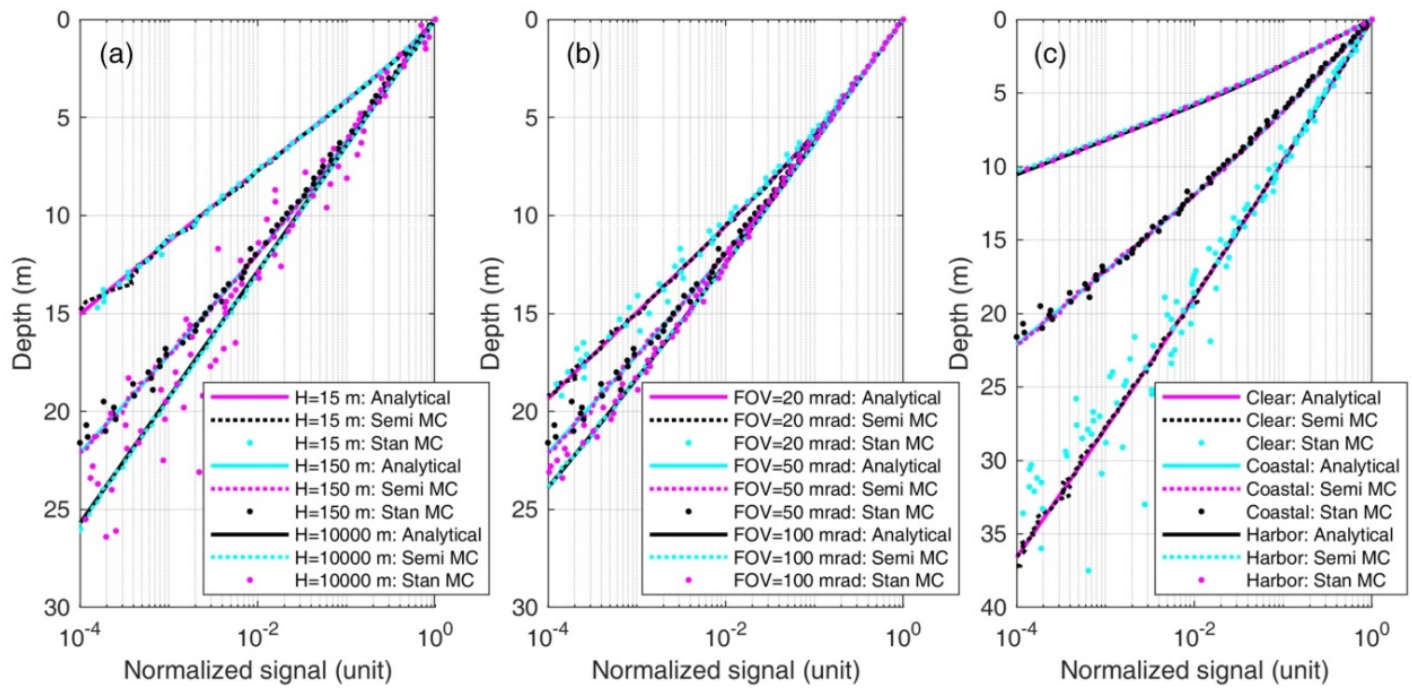

Fig. 2. Normalized lidar signals are calculated by the analytical model, semianalytic MC algorithm and standard MC algorithm at different: (a) heights; (b) FOVs; and (c) water types. 
Figure 3 shows lidar signals calculated by the analytical model (orange solid lines) and measured by the lidar (blue solid lines with error bars) with a large, full FOV of $200 \mathrm{mrad}$. The average values in blue solid lines and standard deviations in blue error bars of 10 lidar-measured signals during the in situ operation period are plotted. Dynamic ranges of 3-4 orders of magnitude can then be realized in most cases. As shown in Fig. 3, the analytical model can perfectly match the lidar-measured results at different stations $[6,9]$.

_ Analytical model _
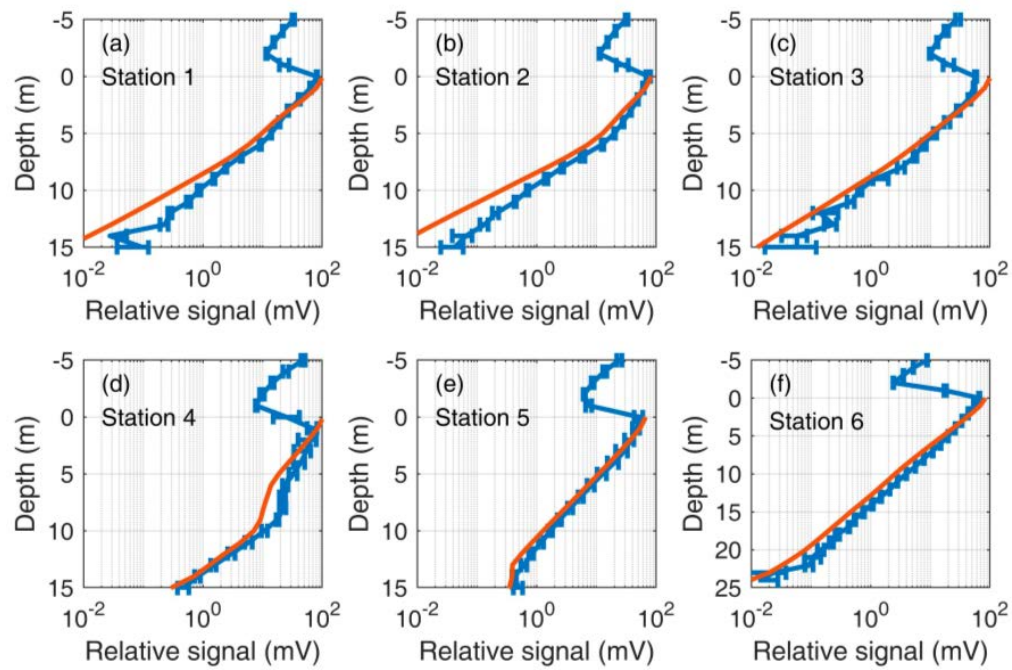

Fig. 3. (a)-(f) are the lidar signals calculated by the analytical model (orange solid lines) and measured by the lidar (blue solid lines with error bars) at Stations 1-6, respectively.

\subsection{Phase function effects}

The effective $180^{\circ}$ VSF $\beta_{p}^{\prime \pi}$ can be obtained from the simulated signals. $\beta_{m}^{\prime \pi}$ is assumed to be equal to true molecular $180^{\circ}$ VSF $\beta_{m}^{\pi}$ because the molecular VSF is smooth in the backward direction. Figures 4(a)-4(c) show the simulation results of Cases A-C, respectively. The true $180^{\circ}$ particulate VSFs $\beta_{p}^{\pi}$ (dashed lines) that are the products of the scattering coefficients and phase functions at $180^{\circ}$ remain constant with depth. The effective $180^{\circ}$ particulate VSFs $\beta_{p}^{\prime \pi}$ (solid lines) typically remain consistent with $\beta_{p}^{\pi}$ at the water surface but deviate from $\beta_{p}^{\pi}$ with increasing depth. The degrees of deviations are closely related to the simulation conditions and phase function [10].
— - OTHG: Effective

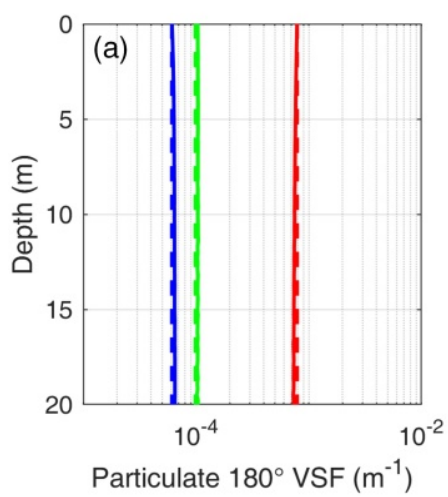

- TTHG: Effective

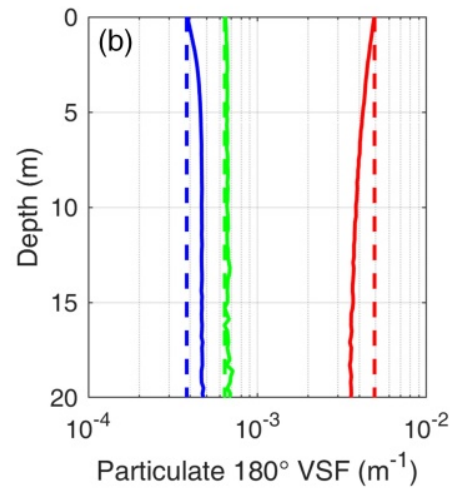

FF: Effective - - FF: True

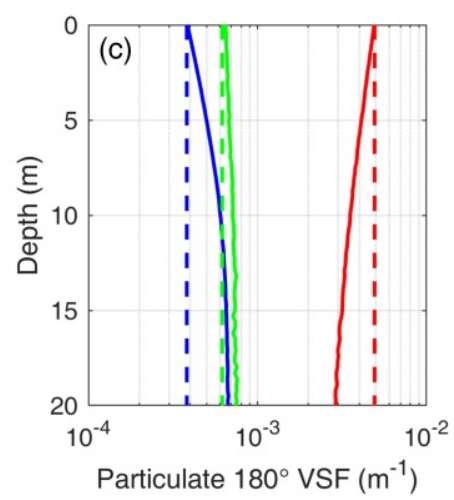

Fig. 4. Phase function effects on the effective particulate $180^{\circ}$ VSF under (a) Case A (clear ocean, FOV of 50 mrad), (b) Case B (coastal ocean, FOV of $50 \mathrm{mrad}$ ) and (c) Case C (coastal ocean, FOV of $200 \mathrm{mrad})$. 
The lidar attenuation coefficient $\alpha$ can be retrieved, as shown in Fig. 5. The sum of the absorption and backscattering coefficients $\left(a+b_{b}\right)$ (black dashed lines) is used as a reference because $\alpha$ should be always greater

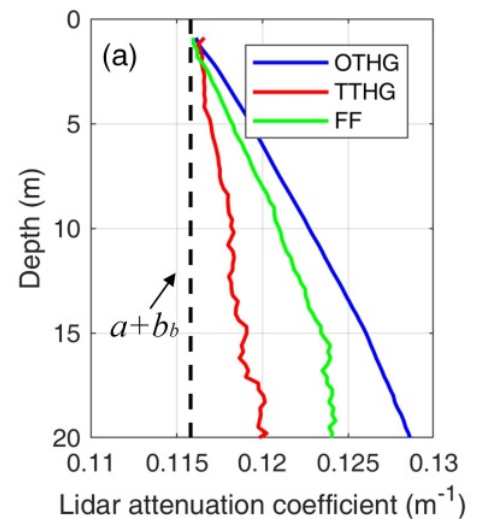

than $\left(a+b_{b}\right)$ under QSA approximation [3, 11]. The term $\alpha$ is close to $\left(a+b_{b}\right)$ for all phase functions at the water surface and increases with depth because of loss during multiple scattering.

Fig. 5. Phase function effects on the lidar attenuation coefficient under (a) Case A (clear ocean, FOV of 50 mrad), (b) Case B (coastal ocean, FOV of $50 \mathrm{mrad}$ ) and (c) Case C (coastal ocean, FOV of $200 \mathrm{mrad}$ ).

\section{ACKNOWLEDGEMENTS}

This work was supported in part by the National Key Research and Development Program of China under Grant 2016YFC1400900 and Grant 2016YFC0200700, in part by the National Natural Science Foundation of China (NSFC) under Grant 41775023 and Grant 61475141, in part by Zhejiang Provincial Natural Science of China under Grant LR19D050001.

\section{REFERENCES}

1. Churnside, J.H., Review of profiling oceanographic lidar. Optical Engineering, 2014. 53(5): p. 051405.

2. Hostetler, C., et al., Spaceborne Lidar in the Study of Marine Systems. Annual review of marine science, 2017: p. 13.1-13.27.

3. Bissonnette, L.R., Lidar and Multiple Scattering. 2005: Springer. 43-103.

4. Wang, L., S.L. Jacques, and L. Zheng, $M C M L-$ Monte Carlo modeling of light transport in multi-layered tissues. Computer methods and programs in biomedicine, 1995. 47(2): p. 131-146.

5. Poole, L.R., D.D. Venable, and J.W. Campbell, Semianalytic Monte Carlo radiative transfer model for oceanographic lidar systems. Applied Optics, 1981. 20(20): p. 3653-6.

6. Zhou, Y., et al., Validation of the Analytical Model of Oceanic Lidar Returns: Comparisons with Monte Carlo Simulations and Experimental Results. Remote Sensing, 2019. 11(16): p. 1870.

7. Katsev, I.L., et al., Efficient technique to determine backscattered light power for various atmospheric and oceanic sounding and imaging systems. Journal of the Optical Society of America A, 1997. 14(6): p. 13381346.

8. Malinka, A.V. and E.P. Zege, Analytical modeling of Raman lidar return, including multiple scattering. Applied optics, 2003. 42(6): p. 1075-1081.

9. Liu, D., et al., Lidar Remote Sensing of Seawater Optical Properties: Experiment and Monte Carlo Simulation. IEEE Transactions on Geoscience and Remote Sensing, 2019. 57(11): p. 9489-9498.

10. Liu, D., et al., Phase function effects on the retrieval of oceanic high-spectral-resolution lidar. Optics Express, 2019. 27(12): p. A654A668.

11. Kopilevich, Y.I. and A. Surkov, Mathematical modeling of the input signals of oceanological lidars. Journal of Optical Technology, 2008. 75(5): p. 321-326. 